Pacific Journal of Mathematics

INVERTING DOUBLE KNOTS

ur CARrington Whiten 


\title{
INVERTING DOUBLE KNOTS
}

\author{
WILBUR WHITTEN
}

\section{We disprove J. Montesinos's conjecture that every inver- tible knot in $S^{3}$ is strongly invertible.}

Let $K$ denote a tame, oriented knot in $S^{3}$, and fix an orientation of $S^{3}$. If there exists an orientation-preserving, PL involution of $S^{3}$ that inverts $K$, then $K$ is strongly invertible. J. Montesinos proposed this definition [5], and he has conjectured [3; Problem 1.6, p. 277] that every invertible knot is strongly invertible. In this paper, we disprove this conjecture; our results are as follows.

THEOREM 1. A knot $K$ is strongly invertible if and only if each double of $K$ is strongly invertible.

Corollary. No double of a noninvertible knot is strongly invertible; hence, there exist invertible knots that are not strongly invertible.

Proof of Corollary. Any double knot is invertible [6; Theorem 1, p. 235].

THEOREM 2. If $L$ is a strongly invertible knot with exactly one maximal companion $C_{L}$, then $C_{L}$ is also strongly invertible.

Section 1 contains a preliminary lemma. We prove Theorems 1 and 2 , in $\S 2$. In $\S 3$, we give a counterexample to the converse of Theorem 2; in $\S 4$, we discuss surgery on invertible knots, give several examples, and formulate a conjecture.

I wish to thank C. Gordon and K. Murasugi for discussing this work with me.

1. Preliminaries. In this paper, all spaces are polyhedrons; the three-sphere has a fixed orientation; all maps are piecewise linear; all submanifolds, polyhedral; and all knots, oriented. We shall need the following lemma.

Positioning Lemma. If $K$ is a strongly invertible knot, then there exists an orientation-preserving, K-inverting, $\mathrm{PL}$ involution $\rho: S^{3} \rightarrow S^{3}$ with nonempty, fixed point set $A$ and there exists a polyhedral, $\rho$-invariant 2-sphere $S$ such that (a) the "axis" $A$ of $\rho$ belongs to $S$, (b) the set $K \cap A$ contains exactly two points, (c) the knot $K$ is transverse with respect to the 2-sphere $S$, and (d) the set $K \cap S$ contains only a finite number of points. 
Proof. Because $K$ is strongly invertible, there exists an orientation-preserving, $K$-inverting, PL involution $\rho^{\prime}: S^{3} \rightarrow S^{3}$. The Lefschetz fixed-point theorem implies that $\rho^{\prime} \mid K$ has exactly two fixed points. Because the fixed-point set (or axis) $A^{\prime}$ of $\rho^{\prime}$ is, therefore, nonempty, the axis $A^{\prime}$ must be a knot [7; Theorem, p. 162]. If we set $S^{3}=$ $R^{3} \cup\{\infty\}$, then, because $\rho^{\prime}$ is piecewise linear and orientation-preserving, there exists a PL autohomeomorphism $\varphi$ of $S^{3}$ such that $\varphi \rho^{\prime} \varphi^{-1}\left(=\rho_{\mathrm{o}}\right)$ is a "standard" (orthogonal) $180^{\circ}$-rotation and such that $\varphi\left(A^{\prime}\right)=(y$-axis $) \cup\{\infty\}\left(=A_{0}\right)[10]$.

Because $\varphi(K)$ is polyhedral, because the rotation $\rho_{0}$ takes $\varphi(K)$ onto itself, and because $\varphi(K)$ meets $A_{0}$ in only two points, $x_{1}$ and $x_{2}$, we can (if necessary) find a small angle $\alpha^{\circ}(\alpha>0)$ such that an $\alpha^{\circ}$-rotation $\rho_{\alpha}$ about $A_{0}$ takes $\varphi(K)$ to a knot $\rho_{\alpha} \varphi(K)$ that is transverse to the 2 -sphere $S_{0}(=(y z$-plane $) \cup\{\infty\})$ at each of the points, $x_{1}$ and $x_{2}$. We shall find a knot $K^{\prime}$ (ambient isotopic to $\rho_{\alpha} \varphi(K)$ ) such that $K^{\prime}$, the involution $\rho_{0}$ (of $S^{3}$ ) with axis $A_{0}$, and the 2 -sphere $S_{0}$ satisfy the hypothesis and the conclusion of the lemma. The lemma's proof will easily follow.

Choose $\varepsilon(>0)$ so that (closed) $\varepsilon$-neighborhood $V_{\varepsilon}$ of $\rho_{\alpha} \varphi(K)$ is a solid torus; such a choice is possible, because $\rho_{\alpha} \varphi(K)$ is polyhedral in $S^{3}$. Because $\rho_{\alpha} \varphi(K)$ is transverse to $S_{0}$ at $x_{1}$ and at $x_{2}$, we can restrict $\varepsilon$ so that $V_{\varepsilon} \cap S_{0}$ contains (among other things) two disjoint meridional disks, $E_{1}$ and $E_{2}$, of $V_{\varepsilon}$, with $E_{i} \cap \rho_{\alpha} \varphi(K)=\left\{x_{i}\right\}(i=1,2)$. By a final restriction of $\varepsilon$, we can assume that $V_{\varepsilon} \cap A_{0}=\left(E_{1} \cup E_{2}\right) \cap$ $A_{0}$ (=two, disjoint arcs). (The constructions involved in our restrictions of $\varepsilon$ are standard, and we shall omit them.) Finally, note that $\rho_{0}\left(V_{\varepsilon}\right)=V_{\varepsilon}$.

The points $x_{1}$ and $x_{2}$ divide $\rho_{\alpha} \varphi(K)$ into two (closed) arcs, $k_{1}$ and $k_{2}$; the disks $E_{1}$ and $E_{2}$ divide $V_{\varepsilon}$ into (closed) 3-cells, $B_{1}$ and $B_{2}$, with $k_{i}$ unknotted in $B_{i}(i=1,2)$ (see [4; p. 134]). We note that $\rho_{0}\left(B_{1}\right)=B_{2}$ and that $B_{i} \cap A_{0}=\left(E_{1} \cup E_{2}\right) \cap A_{0}$.

Keeping $x_{1}$ and $x_{2}$ fixed, we now put $k_{1}$ in general position with respect to $S_{0}$ by an orientation-preserving autohomeomorphism $h_{1}: S^{3} \rightarrow S^{3}$ moving each point of $k_{1}$ less than $\varepsilon$. We can evidently assume that $h_{1} \mid\left(S^{3}-\right.$ Int $\left.B_{1}\right)$ is the identity map.

The arc $\rho_{0} h_{1}\left(k_{1}\right)$ is clearly unknotted in $B_{2}$. Hence, there exists an orientation-preserving autohomeomorphism $h_{2}: S^{3} \rightarrow S^{3}$ taking $k_{2}$ onto $\rho_{0} h_{1}\left(k_{1}\right)$ and leaving each point of $S^{3}-\operatorname{Int} B_{2}$ fixed. The autohomeomorphism $h$ of $S^{3}$ given by

$$
h(x)= \begin{cases}h_{i}(x), & \text { if } x \in \operatorname{Int} B_{i}(i=1,2) \\ x, & \text { otherwise }\end{cases}
$$

preserves the orientation of $S^{3}$ and takes $\rho_{\alpha} \varphi(K)$ onto a knot $h_{1}\left(k_{1}\right) \cup$ 
$\rho_{0} h_{1}\left(k_{1}\right)$ that is in general position with respect to $S_{0}$ and that is strongly inverted by $\rho_{0}$. We set $K^{\prime}=h \rho_{\alpha} \varphi(K)\left(=h_{1}\left(k_{1}\right) \cup \rho_{0} h_{1}\left(k_{1}\right)\right)$ and note that the knot $K^{\prime}$, the involution $\rho_{0}$ with axis $A_{0}$, and the 2sphere $S_{0}$ satisfy the hypothesis and conclusion of the lemma. The proof of the lemma now follows by taking $\rho=\left(h \rho_{\alpha} \varphi\right)^{-1} \rho_{0}\left(h \rho_{\alpha} \varphi\right)$, taking $A=A^{\prime}\left(=\Phi^{-1}\left(A_{0}\right)=\left(h \rho_{\alpha} \varphi\right)^{-1}\left(A_{0}\right)\right)$, and taking $S=\left(h \rho_{\alpha} \varphi\right)^{-1}\left(S_{0}\right)$.

\section{Proofs.}

Proof of Theorem 1. We shall assume that $K$ is not trivial, for otherwise, the theorem is evidently true.

(1) Necessity. We assume that $K$ is strongly invertible. Let $\rho$, and $A$, and $S$ denote the objects our Positioning Lemma guarantees, and let $K \cap A=\left\{x_{1}, x_{2}\right\}$. By the Positioning Lemma's proof, we can assume (without loss of generality) that $\rho$ is the $180^{\circ}$-rotation about $A(=(y$-axis $) \cup\{\infty\})$ and that $S=(y z$-plane $) \cup\{\infty\}$. Moreover, we can choose $\varepsilon(>0)$ and $V_{\varepsilon}$ exactly as in the lemma's proof. We have $K=$ $k_{1} \cup k_{2}$ (with $\rho\left(k_{1}\right)=k_{2}$ ) and $V_{\varepsilon}=B_{1} \cup B_{2}$; moreover, $E_{i} \cap A(i=1,2)$ is a properly imbedded arc in $E_{i}$.

Let $C$ denote a cylindrical 3-cell with core $k$ and with two disks, $D_{1}$ and $D_{2}$, meeting in an arc and imbedded in $C$, as shown in Figure 1. Let $v$ be a (closed) arc in $\operatorname{Int}\left(E_{2} \cap A\right)$ such that $x_{2} \in \operatorname{Int}(v)$ (see Figure 2(a)). It is easy to find an arc $v_{1} \subset \operatorname{Int} E_{1}$ such that $v_{1} \cap A=$ $\left\{x_{1}\right\}=v_{1} \cap \rho\left(v_{1}\right)=A \cap \operatorname{Int} v_{1}$; note that $\rho\left(v_{1}\right) \subset \operatorname{Int} E_{1}$ (see Figure $2(\mathrm{~b})$ ).

Now, let $g: C \rightarrow B_{1}$ be a homeomorphism such that $g\left(E_{i}^{\prime}\right)=E_{i}(i=$ $1,2)$, such that $g\left(v_{1}^{\prime}\right)=v_{1}$ and $g\left(v_{2}^{\prime}\right)=\rho\left(v_{1}\right)$, such that $g\left(v^{\prime}\right)=v$, and such that $g(k)=k_{1}$. Then $\left[g\left(D_{1} \cup D_{2}\right)\right] \cup\left[\rho g\left(D_{1} \cup D_{2}\right)\right]$ is a singular disk $\Sigma$ with one clasping singularity, and the $\partial \Sigma$ is a double of $K$ with twisting number $\sigma$ (an integer depending on the homeomorphism $\left.g: C \rightarrow B_{1}\right)$ and with self-intersection number $\eta(= \pm 2)$. (By changing $g$ (to change $\sigma$ ) and by replacing $C$ with its mirror image (to change the sign of $\eta$ ), we can assume that $\partial \Sigma$ is any double of $K$ that we desire.) Evidently, $\partial \Sigma$ is strongly invertible (by the involution $\rho$ ). This completes the proof of the necessity.

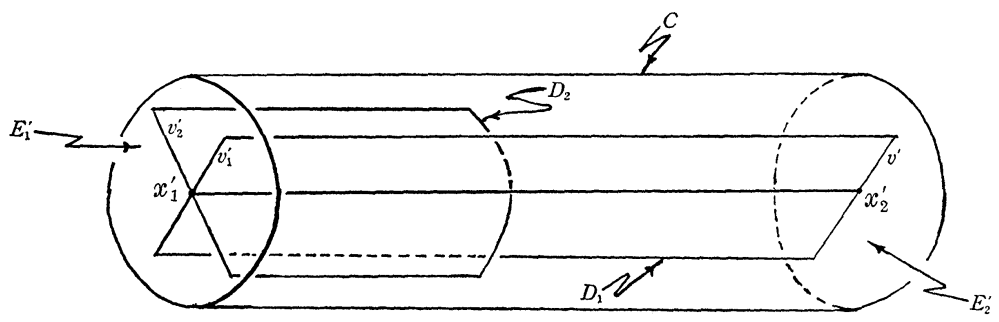

Figure 1 


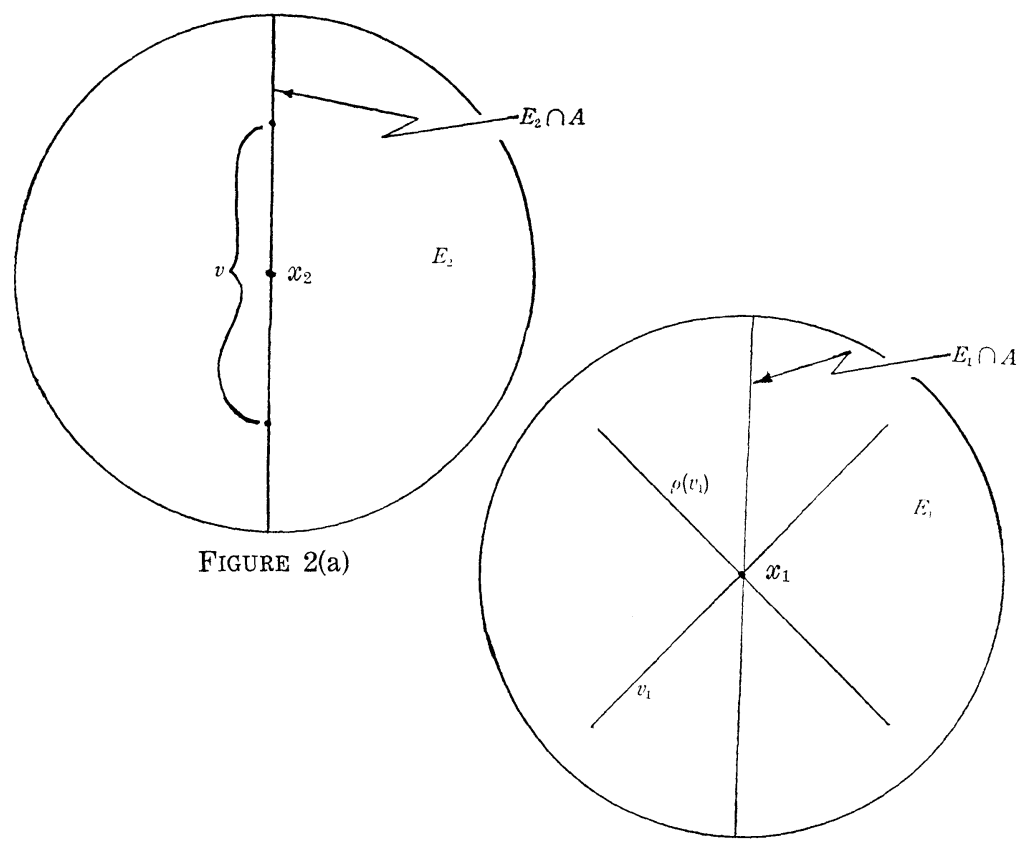

FIGURE 2(b)

(2) Sufficiency. We assume that some double, $D_{K}$, of $K$ is strongly invertible. Replace $K$ by $D_{K}$ in the Positioning Lemma; we can assume that $\rho$ is the standard rotation (of period 2) about $A(=(y$-axis $) \cup\{\infty\})$, that $S=(y z$-plane $) \cup\{\infty\}$, and that $D_{K} \cap A=\left\{x_{1}, x_{2}\right\}$.

Let $V^{*}$ denote a (closed) regular neighborhood of a clasping disk whose boundary is $D_{K}$; note that $K$ is equivalent to a core of $V^{*}$ [6; p. 238]. Now $K$ is a unique maximal companion $D_{K}$ [6; p. 242]; that is, any companion of $D_{K}$, other than $K$, is also a companion of $K$. Hence, the torus $\rho\left(\partial V^{*}\right)$ is ambient isotopic to $\partial V^{*}$ in $S^{3}-D_{K}$. So, by [9; Theorem 1, p. 223], the $\partial V^{*}$ is ambient isotopic (in $S^{3}-D_{K}$ ) to a torus $T$ in general position with respect to $A$, and either $\rho(T) \cap$ $T=\varnothing$ or $\rho(T)=T$. If $\rho(T) \cap T=\varnothing$, then $T$ and $\rho(T)$ are parallel. Because $\rho^{2}(T)=T$ and because each of $\rho(T)$ and $T$ separates $S^{3}-D_{K}$, it easily follows that $\rho$ moves fixed points of itself, which is absurd. Thus, $\rho(T)=T$.

Now $T$ splits $S^{3}$ into a solid torus $V$ (containing $D_{K}$ in its interior) and a $K$-knot manifold. If $A \cap T=\varnothing$, then $A \subset \operatorname{Int} V$, because $A \cap$ $D_{K} \neq \varnothing$. Because $K$ is knotted and $A$ is unknotted, $A$ belongs to a polyhedral 3-cell $\subset$ Int $V$; otherwise, $A$ would have a companion, which it does not [6]. Applying Tollefson's lemma [8; Lemma 1, p. 141], we can find a 2-sphere $S^{\prime} \subset \operatorname{Int}(V-A)$ such that $S^{\prime}$ bounds no 3-cell in $V-A$ and such that either $\rho\left(S^{\prime}\right) \cap S^{\prime}=\varnothing$ or $\rho\left(S^{\prime}\right)=S^{\prime}$. As with the tori $T$ and $\rho(T)$ in the preceding paragraph, we cannot 
have $\rho\left(S^{\prime}\right) \cap S^{\prime}=\varnothing$. If $\rho\left(S^{\prime}\right)=S^{\prime}$, then take the 3-cell $B^{3}\left(\subset S^{3}\right)$ that does not contain $A$ and that $S^{\prime}$ bounds (in $S^{3}$ ), and consider the homeomorphism $\rho \mid B: B \rightarrow B$. By the Brouwer fixed-point theorem, $\rho \mid B$ has a fixed point, and so $\rho$ has a fixed point not on $A$ (which it does not). Hence, $A \cap T \neq \varnothing$.

Because $T$ is in general position with respect to $A$, the cardinality $b$ of $A \cap T$ is finite. Let $T_{0}$ denote the orbit space of $\rho \mid T$. The projection $p: T \rightarrow T_{0}$ is a branched covering, and the two Euler characteristics, $\chi(T)$ and $\chi\left(T_{0}\right)$, are related by the Riemann-Hurwitz branch-point formula,

$$
\chi(T)=2 \chi\left(T_{0}\right)-b ;
$$

see $[1 ; p, 93]$. But $\chi(T)=0$ and $b>0$. Hence, $\chi\left(T_{0}\right)=2$, and so $T_{0}$ is a 2-sphere and $b=4$. (Because the orbit space of $\rho$ is $S^{3}$ and because $S^{3}$ contains no projective planes, we cannot have $\chi\left(T_{0}\right)=1$.)

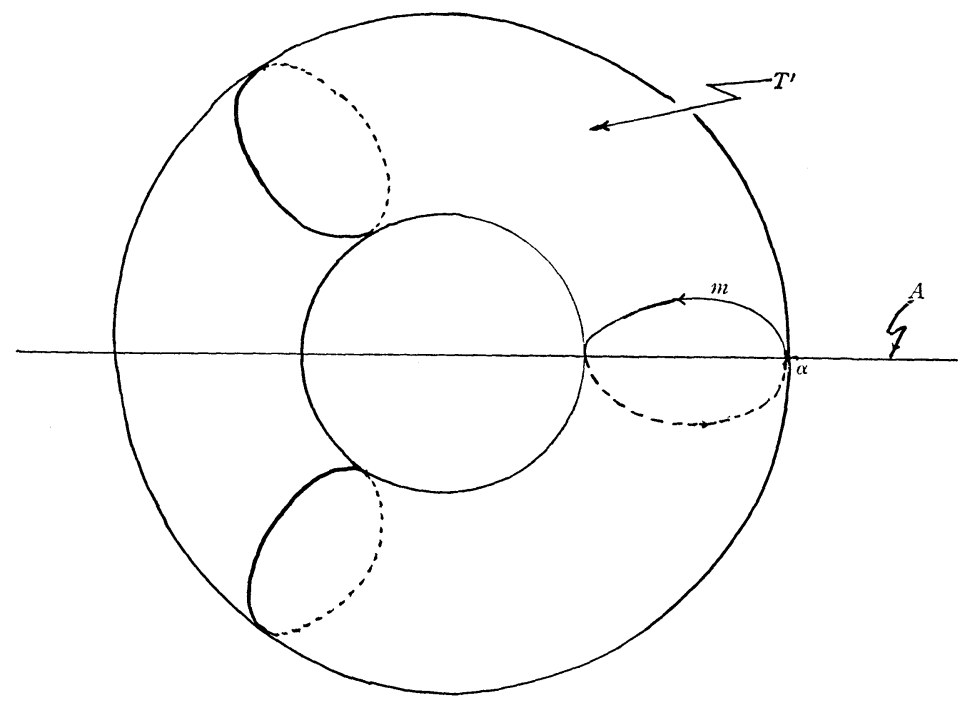

Figure 3

Now let $T^{\prime}$ denote the torus $(r-2)^{2}+z^{2}=1$ (see Figure 3), let $m$ denote the curve $\left\{(r, z) \mid \theta=0\right.$ and $\left.(r-2)^{2}+z^{2}=1\right\}$ (which we shall take as one of the two components of $\left.T^{\prime} \cap S\right)$, and let $K_{(\bar{p}, \bar{q})}((\bar{p}, \bar{q})=$ 1) denote the torus knot $\{(r, z) \mid r=2+\cos (\bar{p} \theta / \bar{q}), z=\sin (\bar{p} \theta / \bar{q})\}$ on $T^{\prime}$ (cf. [2; p. 92]). To fix the $(r, \theta, z)$-coordinate system on $T^{\prime}$, let the point $\alpha$ shown in Figure 3 have $(r, \theta, z)$-coordinates $(3,0,0)$. Note that $\rho\left(T^{\prime}\right)=T^{\prime}$ and that $\rho(m)=m^{-1}$ (after we have oriented $m$ ). If $T_{0}^{\prime}$ denotes the orbit space of $\rho \mid T^{\prime}$, then the projection $p^{\prime}: T^{\prime} \rightarrow T_{0}^{\prime}$ is a branched covering. As with $p: T \rightarrow T_{0}$, the covering $p^{\prime}$ has four branch points, and $T_{0}^{\prime}$ is a 2 -sphere. 
According to $\left[1\right.$; Theorem 3.4, p. 94], the coverings $p$ and $p^{\prime}$ are equivalent; that is, there exist homeomorphisms $\psi: T \rightarrow T^{\prime}$ and $\gamma: S^{2} \rightarrow S^{2}$ such that $p^{\prime} \psi=\gamma \rho$. It follows easily that $\varphi$ preserves covering fibers.

Thus, if $\{x, \rho(x)\}$ is a fiber of $p$, then $\{\psi(x), \psi \rho(x)\}$ is a fiber of $p^{\prime}$, and so $\left(\rho \mid T^{\prime}\right) \psi(\rho \mid T)(x)=\psi(x)$; that is, $\psi=\left(\rho \mid T^{\prime}\right) \psi(\rho \mid T)$. Because $\rho^{2}=i d$., we have $\left(\rho \mid T^{\prime}\right) \psi=\psi(\rho \mid T)$. Notice that $\rho\left(K_{(\bar{p}, \bar{q})}\right)=K_{(\bar{p} \bar{q})}^{-1}$; thus, for any $(\bar{p}, \bar{q})$-torus knot, there exists a representative, $K_{(\bar{p} \bar{q})}$, of it on $T^{\prime}$ that $\rho$ inverts (and, hence, strongly inverts).

If $\lambda$ is an (oriented) longitude of $K$ on $T$, then $\psi(\lambda)$ is isotopic on $T^{\prime}$ to $m$ or to one of the torus knots $K_{\left(\bar{p}_{1}, \bar{q}_{1}\right)}$, for some pair $\left(\bar{p}_{1}, \bar{q}_{1}\right)$. Thus, either $\psi^{-1}(m)$ or $\psi^{-1}\left(K_{\left(\overline{p_{1}}, \bar{q}_{1}\right)}\right)$ is a longitude of $T$ meeting the axis $A$ of $p$ in exactly two points, because $\psi$ maps branch points of $p$ to branch points of $p^{\prime}$. Because $\left(\rho \mid T^{\prime}\right) \psi=\psi(\rho \mid T)$, we have either $\rho\left(\psi^{-1}(m)\right)=\psi^{-1}\left(\rho \mid T^{\prime}\right)(m)=\psi^{-1}\left(m^{-1}\right)=\left[\psi^{-1}(m)\right]^{-1}$ or, similarly, $\rho\left(\psi^{-1}\left(K_{\left(\bar{p}_{1}, \bar{q}_{1}\right)}\right)\right)=\left[\psi^{-1}\left(K_{\left(\bar{p}_{1}, \bar{q}_{1}\right)}\right)\right]^{-1}$. Therefore, $\rho$ strongly inverts a longitude of $K$, and it follows that $K$ itself is strongly invertible.

Proof of Theorem 2. We need only note that, in the proof of Theorem 1, the sufficiency portion depends on the uniqueness of the maximal companion $K$ of $D_{K}$ and not on the knot type of $D_{K}$.

3. A counterexample. The noninvertible knot $\mathscr{K}$ in $[11$; Figure 3, p. 1275] is a counterexample to the converse of Theorem 2. Because the knots $3_{1}$ and $5_{1}$ (of the Alexander-Briggs table) are simple, one can apply Schubert's theorem [6; p. 216] to show that $\mathscr{K}$ has exactly one maximal companion, which is a trefoil knot and, hence, strongly invertible; details of the application are routine, and we shall omit them.

4. A conjecture. A link $L$ in $S^{3}$ is strongly invertible, if there exists an orientation-preserving $\mathrm{PL}$ involution of $S^{3}$ that inverts each component of $L$. In [5, Theorem 1, p. 231], Montesinos proved that any 3-manifold derived from surgery on a strongly invertible link is a 2-fold cyclic covering space of $S^{3}$ branched over a link and, conversely, that one can produce any particular 2 -fold branched cyclic covering space of $S^{3}$ by surgery on a suitable, strongly invertible link. I do not know whether nontrivial surgery on a knot that is not strongly invertible will produce a 2 -fold branched cyclic covering space of $S^{3}$. It is, however, a different story for links. Here are some examples.

F. González-Acuña and J. Montesinos gave the first such examples (unpublished). Assign any rational coefficient to the componen't $K_{1}$ of the unsplittable and noninvertible Borromean rings, $K_{1} \cup K_{2} \cup K_{3}$ 
[5]. Take nonzero integers, $a$ and $b$, and assign the coefficient $1 / a$ to $K_{2}$ and the coefficient $1 / b$ to $K_{3}$. We now have a surgical description of a closed, connected, orientable 3-manifold, $M$. By applying an appropriate twist across a disk spanning each of $K_{2}$ and $K_{3}$, we can replace our original surgical description on $M$ by one involving only a knot, $K$, which (with a little adjusting) is easily seen to be strongly invertible. Hence, $M$ is a 2 -fold branched cyclic covering space of $S^{3}$. Some of the various knots that $K$ might be are $8_{3}, 10_{3}$, and any twist knot.

For the second group of examples, let $K_{1}$ denote a double of a noninvertible knot and let $K_{2}$ denote a trivial knot in $S^{3}-K_{1}$ placed near the "critical" part of $K_{1}$ so that exactly one (suitable) twist, $t$, across a disk spanning $K_{2}$, will unknot $K_{1}$. Now assign any rational coefficient to $K_{1}$ and assign either +1 or -1 to $K_{2}$ so that the coefficient of $K_{2}$ becomes $\infty$ after the twist $t$. The link $K_{1} \cup K_{2}(=L)$ is invertible, but not strongly invertible. Furthermore, with the two coefficients attached, $L$ provides a surgical description of a manifold $N$. After twisting by $t$ about a disk spanning $K_{2}$, we can replace our first surgical description of $N$ by one involving only a trivial knot. Hence, $N$ is a 2 -fold branched cyclic covering space of $S^{3}$; in fact, $N$ is a lens space.

CONJECTURE. No manifold obtained from nontrivial surgery on a double of a noninvertible knot is a 2-fold branched cyclic covering space of $S^{3}$.

We conclude with two remarks, added in October, 1980, just before the paper went to press.

REMARK 1. Let $K$ be a knot nontrivially imbedded in the interior of an unknotted solid torus $V$ in $S^{3}$, and suppose that one can invert $K$ inside $V$ (without disturbing $S^{3}-\operatorname{Int}(V)$ ). Let $W$ be a solid torus in $S^{3}$ whose core is not strongly invertible, and let $f: V \rightarrow W$ be a faithful homeomorphism. With only minor technical restrictions on $K$, we can conclude that $f(K)$ is invertible but not strongly (see Theorem 2 of [12]). One can easily construct examples (each with genus $>1$ ) that are not double knots (see [12]).

REMARK 2. Richard Hartley has independently constructed counterexamples to Montesinos's conjecture (that every invertible knot is strongly invertible); see Hartley's paper [Knots and involutions, Math. Zeit., 171 (1980), 175-185]. 


\section{REFERENCES}

1. I. Bernstein and A. L. Edmonds, On the construction of branched coverings of lowdimensional manifolds, Trans Amer. Math. Soc., 247 (1979), 81-124.

2. R. H. Crowell and R. H. Fox, Introduction to Knot Theory, Graduate Texts in Mathematics 57, Springer-Verlag, New York, 1977.

3. R. Kirby, Problems in low dimensional manifold topology, Proceeding of Synposia in Pure Mathematics XXXII, Amer. Math. Soc., Providence, R. I., 1978.

4. E. E. Moise, Geometric Topology in Dimensions 2 and 3, Graduate Texts in Mathematics 47, Springer-Verlag, New York, 1977.

5. J. Montesinos, Surgery on links and double branched covers of $\mathrm{S}^{3}$; Knots, Groups, and 3-Manifolds, Annals of Mathematics Studies 84, 227-259, Princeton University Press, Princeton, N. J., 1975.

6. H. Schubert, Knoten und Vollringe, Acta Math., 90 (1953), 131-286.

7. P. A. Smith, Transformations of finite period, Ann. of Math., 39 (1938), 127-164.

8. J. L. Tollefson, Involutions on $S^{1} \times S^{2}$ and other 3-manifolds, Trans. Amer. Math. Soc., 183 (1973), 139-152.

9. - Periodic homeomorphisms of 3-manifolds fibered over $S^{1}$, Trans. Amer. Math. Soc., 223 (1976), 223-234.

10. F. Waldhausen, Über Involutionen der 3-sphäre, Topology, 8 (1969), 81-91.

11. W. Whitten, Surgically transforming links into noninvertible knots, Amer. J. Math., 44 (1972), 1269-1281.

12. - Switching and inverting knots, preprint.

Recrived January 24, 1979.

UNIVERSiTY OF SOUTHWESTERN LOUISIANA

LAFAYETTE, LA 70504 


\section{PACIFIC JOURNAL OF MATHEMATICS}

\section{EDITORS}

DONALD BABBITT (Managing Editor)

University of California

Los Angeles, California 90024

Hugo RossI

University of Utah

Salt Lake City, UT 84112

C. C. MOORE and ANDREW OGG

University of California

Berkeley, CA 94720

\section{J. DugundJI}

Department of Mathematics University of Southern California Los Angeles, California 90007

R. Finn and J. Milgram Stanford University

Stanford, California 94305

\section{ASSOCIATE EDITORS}
R. ARENS
E. F. BeCKENBACH
B. H. Neumann
F. WoLF
K. YoSHIDA

\section{SUPPORTING INSTITUTIONS}

UNIVERSITY OF ARIZONA

UNIVERSITY OF BRITISH COLUMBIA

CALIFORNIA INSTITUTE OF TECHNOLOGY

UNIVERSITY OF CALIFORNIA

MONTANA STATE UNIVERSITY

UNIVERSITY OF NEVADA, RENO

NEW MEXICO STATE UNIVERSITY

OREGON STATE UNIVERSITY
UNIVERSITY OF OREGON

UNIVERSITY OF SOUTHERN CALIFORNIA

STANFORD UNIVERSITY

UNIVERSITY OF HAWAII

UNIVERSITY OF TOKYO

UNIVERSITY OF UTAH

WASHINGTON STATE UNIVERSITY

UNIVERSITY OF WASHINGTON 


\section{Pacific Journal of Mathematics}

\section{Vol. 97, No. $1 \quad$ January, 1981}

Charles A. Asmuth and Joe Repka, Tensor products for $S L_{2}(\mathfrak{k})$. II.

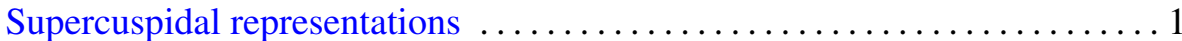

Joseph Barback, On finite sums of regressive isols . ................. 19

Matthew G. Brin and Daniel Russell McMillan, Jr., Generalized

three-manifolds with zero-dimensional nonmanifold set ............29

Kun Soo Chang, Converse measurability theorems for Yeh-Wiener space . . . 59

Christopher Brian Croke, A "maximal torus" type theorem for complete

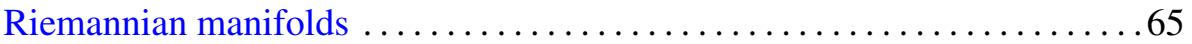

Gustave Adam Efroymson, Sums of squares in planar Nash rings . . . . . . 75

John Robert Fisher, Axiomatic radical and semisimple classes of rings . . . .81

Betty Kvarda, Consecutive integers for which $n^{2}+1$ is composite .......93

Roosevelt Gentry, New diagram proofs of the Hausdorff-Young theorem

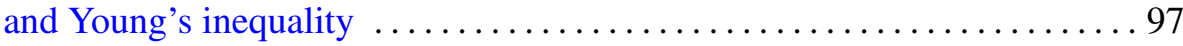

Patrick M. Gilmer, Topological proof of the $G$-signature theorem for $G$

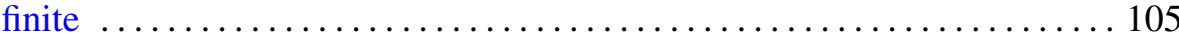

Chung Wei Ha, A noncompact minimax theorem .................. 115

James J. Hebda, Manifolds admitting taut hyperspheres ................ 119

Takayuki Kawada, Sample functions of Pólya processes ............. 125

Peter K. F. Kuhfittig, Common fixed points of nonexpansive mappings by

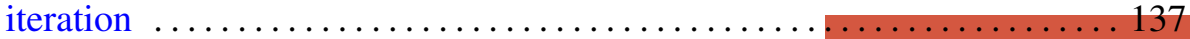

James Thomas Loats and Judith Roitman, Almost rigid Hopfian and dual

Hopfian atomic Boolean algebras .......................... 141

Roger McCann, On embedding semiflows into a radial flow on $l_{2} \ldots \ldots \ldots 151$

John McDonald, Closed orbits of convex sets of operators on the disk

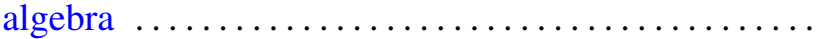

Mark D. Meyerson, Convexity and the table theorem .............. 167

Arnold William Miller, Generic Souslin sets . . . . . . . . . . . . . . . . 171

Takemi Mizokami, On the closed images of paracomplexes $\ldots \ldots \ldots \ldots \ldots 3$

Jagannadham Venkata Pakala and Thomas Stephen Shores, On

compactly packed rings $\ldots \ldots \ldots \ldots \ldots \ldots \ldots \ldots \ldots$

Andrew Pletch, Strong completeness in profinite groups

Wilbur Carrington Whitten, Inverting double knots

James Juei-Chin Yeh, Existence of strong solutions for stochastic

differential equations in the plane 